Pacific Journal of Mathematics

CLASSICAL LINK INVARIANTS AND THE BURAn 


\section{CLASSICAL LINK INVARIANTS AND THE BURAU REPRESENTATION}

\section{DAVID M. GoldSCHMIDT}

The object of this paper is to show how to use the Burau representation of the Artin braid group to calculate some invariants of an oriented link in $\mathbb{S}^{3}$. More precisely, we obtain

(a) generators and relations for the Alexander module, and

(b) a unimodular $(-t)$-Hermitian form on the torsion submodule of the Alexander module (see below for a precise statement). Scaling our form by $\left(1-t^{-1}\right)$ yields a Hermitian form which, for knots, is probably the Blanchfield form. If so, it would then follow from Trotter that the $S$-equivalence class of the Seifert form of a knot can be computed from the Burau representation. Even if this form is the Blanchfield form for knots, the situation for links is less clear because $\left(1-t^{-1}\right)$ need not be invertible in the endomorphism ring of the Alexander module.

Introduction. To state the results precisely, let $B_{n}$ be the $n$-string braid group, let $R=\mathbb{Z}\left[t, t^{-1}\right]$, and let $V_{n}$ be a free $R$-module of rank $n$ affording the unreduced ${ }^{1}$ Burau representation. For $\gamma \in B_{n}$, let $\hat{\gamma}$ be the link in $\mathbb{S}^{3}$ obtained by identifying the ends of a geometrical realization of $\gamma$, and set $W(\gamma)=(1-\gamma)\left(V_{n}\right)$.

THEOREM 1. $V_{n} / W(\gamma)$ depends only on $\hat{\gamma}$. In fact, it is the Alexander module of the disjoint union of $\hat{\gamma}$ with the unknot. Let $U_{n} \subseteq$ $V_{n}$ afford the reduced Burau representation. Then $W(\gamma) \subseteq U_{n}$ and $U_{n} / W(\gamma)$ is the Alexander module of $\hat{\gamma}$.

The fact that the Burau representation is intimately connected with the Alexander module is well known (cf. [1], p. 122) but the exact details may not have appeared previously.

To state Theorem 2 , we let $\mathbb{Q}(t)$ be the field of rational functions and let ${ }^{*}$ be the automorphism of $R$ defined by $t^{*}=t^{-1}$. If $M$ and $N$ are $R$-modules, a $(-t)$-Hermitian form on $M$ with values in $N$ is an $R$-module map $f: M \otimes_{R} M \rightarrow N$ such that $f(x \otimes y)=-t f(y \otimes x)^{*}$. Such a map induces a natural map $M \rightarrow \operatorname{Hom}_{R}(M, N)$. When this map is an isomorphism, $f$ is sometimes called a "perfect pairing".

\footnotetext{
${ }^{1}$ It is essential to use the unreduced Burau representation here.
} 
In the following, $M$ is the $R$-torsion submodule of the Alexander module, and $N$ is $\mathbb{Q}(t) / R$.

THEOREM 2. Let $\bar{A}=\bar{A}(\gamma)$ be the R-torsion submodule of $U_{n} / W(\gamma)$. Then there is a $(-t)$-Hermitian form defined on $\bar{A}(\gamma)$ with values in $\mathbb{Q}(t) / R$ depending only on $\hat{\gamma}$. When the Alexander polynomial is nonzero, the form is a perfect pairing.

The paper is organized as follows. In $\S 2$ we recall the definition of the Burau representation and define an invariant sesqui-linear form which is essentially due to Squier [3]. In $\S 3$ we prove Theorem 1 . In $\S 4$ we prove Theorem 2 by defining, for any braid $\gamma$, a $(-t)$-Hermitian form on $\bar{A}(\gamma)$ which we then show is invariant up to isomorphism under the Markov moves ([1], p. 51). We defer the proof that the form is unimodular (i.e. a perfect pairing) when the Alexander polynomial is non-zero to $\S 5$, in which we describe an algorithm for calculating the form and we do the calculations for the figure eight knot. In $\S 6$ we study the effect of the orientation-reversing symmetries. In $\S 7$ we show how to get a rational-valued form (integral when $\Delta$ is monic) by taking the trace. Finally, in $\S 8$ we apply the results to the $(n, m)$ torus link. We obtain a presentation for the Alexander module as a direct sum of cyclic submodules, and explicit formulae for the (Blanchfield?) form.

2. The Burau representation. Let $B_{n}$ be the $n$-string Artin braid group with standard generators $\sigma_{1}, \sigma_{2}, \ldots, \sigma_{n-1}$, and let $F_{n+1}$ be the free group on $n+1$ free generators $x_{0}, x_{1}, \ldots, x_{n}$. Let $R=$ $\mathbb{Z}\left[t, t^{-1}\right]$. Then $B_{n}$ acts on $F_{n+1}$ via

$$
\sigma_{i}\left(x_{j}\right)= \begin{cases}x_{i} x_{i+1} x_{i}^{-1} & \text { if } j=i \\ x_{i} & \text { if } j=i+1 \\ x_{j} & \text { otherwise. }\end{cases}
$$

Note that $x_{0}$ is fixed by $B_{n}$. Define $\varepsilon: F_{n+1} \rightarrow \mathbb{Z}$ via $\varepsilon\left(x_{i}\right)=1$ for all $i$, and let $K=\operatorname{ker} \varepsilon$. Then $V_{n}=K / K^{\prime}$ is a free $R$-module with basis $f_{i}=x_{0} x_{i}^{-1} K^{\prime} \quad(1 \leq i \leq n)$, where the action of $t$ is given by $t f_{i}=x_{j} f_{i} x_{j}^{-1}$ for any $j$ (one verifies that this is well defined modulo $K^{\prime}$ ). An easy calculation then shows that

$$
\sigma_{i}\left(f_{j}\right)= \begin{cases}(1-t) f_{i}+t f_{i+1} & \text { if } j=i \\ f_{i} & \text { if } j=i+1 \\ f_{j} & \text { otherwise. }\end{cases}
$$


$V_{n}$ affords the (unreduced) Burau representation of $B_{n}$. The vector $u_{n}=\sum_{i=1}^{n} t^{i-1} f_{i} \in V_{n}$ is fixed by $B_{n}$ and so is the augmentation map $\varepsilon_{0}\left(f_{i}\right)=1 \quad(1 \leq i \leq n)$ (not to be confused with its cousin defined on the free group). Let $U_{n}=\operatorname{ker}\left(\varepsilon_{0}\right)$. Then $U_{n}$ has the basis $e_{i}=f_{i}-f_{i+1} \quad(1 \leq i<n)$ and affords the so-called "reduced" Burau representation. An important point which may not have been thoroughly appreciated heretofore is that $U_{n}$ is not a summand of $V_{n}$ as a $B_{n}$-module; in fact $\left\langle u_{n}\right\rangle \oplus U_{n}$ has index $\varepsilon_{0}\left(u_{n}\right)=\sum_{i=1}^{n} t^{i-1}$ in $V_{n}$.

The usual geometric interpretation is to let $B_{n}$ act via the mapping class group on the $(n+1)$-punctured disk $\mathbb{D}_{n+1}$ with $F_{n+1}=\pi_{1}\left(\mathbb{D}_{n+1}\right)$. $K$ is then the fundamental group of an infinite cyclic cover $C$ of $\mathbb{D}_{n+1}$ which can be embedded in $\mathbb{R}^{3}$ as the "parking garage": an infinite vertical stack of $(2 n+2)$-gons with $n+1$ ramps going between successive levels as in Figure 1. Consequently, there is an integer pairing on $H_{1}(C)=K / K^{\prime}$, where $(x, y)_{0}$ is the linking number of the push-off of $x$ with $y$. We define

$$
(x, y)=\sum_{i} t^{i}\left(t^{i} x, y\right)_{0}
$$

Then

$$
\left(f_{i}, f_{j}\right)=\left\{\begin{array}{ll}
1+t & \text { if } i=j, \\
t & \text { if } i<j, \\
1 & \text { if } i>j,
\end{array} \quad\left(e_{i}, e_{j}\right)=\left\{\begin{array}{cl}
1+t & \text { if } i=j \\
-1 & \text { if } i=j-1, \\
-t & \text { if } i=j+1, \\
0 & \text { if }|i-j|>1 .
\end{array}\right.\right.
$$

If ${ }^{*}$ is the automorphism of $\mathbb{Z}\left[t, t^{-1}\right]$ defined by $t^{*}=t^{-1}$, one checks that $(x, \alpha y+z)=\alpha(x, y)+(x, z)$, and $(x, y)=t(y, x)^{*}$. An easy calculation shows that this form is invariant under the action of $B_{n}$. Up to a scale factor and the change of variable $s^{2}=t$, the restriction of this form to $U_{n}$ was discovered by Squier [3].

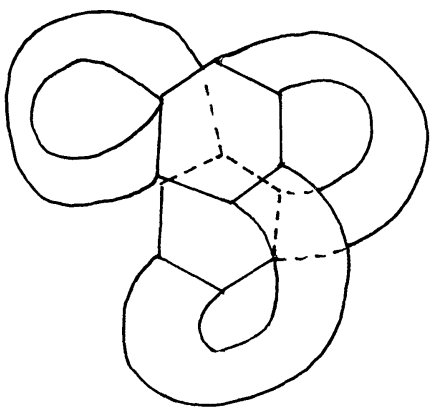

FIGURE 1 
3. The Alexander module. Let $\gamma \in B_{n}$, let $g$ be the union of the geometrical realization of $\gamma$ with an additional straight string, and let $\hat{g}$ be the corresponding link. $\hat{g}$ is the disjoint union of the link defined by $\gamma$ with the unknot. The reason for introducing the unknot will be clarified shortly.

Geometrically, it is advantageous to imagine the passage from $g$ to $\hat{g}$ as occurring in two steps. First, we embed the braid $g$ in a solid torus $T$ and identify the ends in such a way that a parametrization of the resulting path(s) has positive longitudinal derivative at all points. Then we attach a second solid torus along the boundary of $T$ to obtain $\mathbb{S}^{3}$ in the usual way. Let $F=F_{n+1}$. Since the complement $T-g$ is a twisted product of the circle with the $(n+1)$-punctured disk, it's not hard to see that the fundamental group of $T-g$ is the semi-direct product $G=\langle\gamma\rangle F$. When we attach the other torus, the effect on $\pi_{1}$ is to set $\gamma=1$, and thus $\pi_{1}\left(\mathbb{S}^{3}-\hat{g}\right)=G /[\gamma, G]\langle\gamma\rangle \cong F /[\gamma, F]$, where $[\gamma, F]=\left\langle\gamma x \gamma^{-1} x^{-1} \mid x \in F\right\rangle$ (see [1], p. 46 for details).

Let $\varphi: F \rightarrow F /[\gamma, F]$ be the natural map, and let $K=\operatorname{ker}(\varepsilon) \subseteq F$. $K$ defines the "parking garage" whose homology affords the unreduced Burau representation of $B_{n}$. Since $\varepsilon\left(\gamma x \gamma^{-1}\right)=\varepsilon(x)$ it follows that $[\gamma, F] \subseteq K$. Thus, $\varepsilon$ factors through $\varphi$ and defines a map $\hat{\varepsilon}$ : $\pi_{1}\left(\mathbb{S}^{3}-\hat{g}\right) \rightarrow \mathbb{Z}$. $\hat{\varepsilon}(x)$ is just the linking number of $x$ with $\hat{g}$. Consequently, $\varphi(K)$ defines the infinite cyclic cover of $\mathbb{S}^{3}-\hat{g}$ whose homology is the Alexander module. In particular, we see that $K / K^{\prime}[\gamma, F]$ is isomorphic to the Alexander module of $\hat{g}$.

We would like to replace $[\gamma, F]$ by $[\gamma, K]$ because in additive notation the group $K / K^{\prime}[\gamma, K]$ is just $V_{n} /(1-\gamma)\left(V_{n}\right)$. Indeed, since $\gamma$ centralizes $F / K$ it is tempting to conclude that $[\gamma, F]=[\gamma, K]$, but unfortunately this is not in general true. If, however, $F=K X$ where $[\gamma, X]=1$ then the general identity

$$
[\gamma, k x]=[\gamma, k] k[\gamma, x] k^{-1}
$$

implies immediately that $[\gamma, F]=[\gamma, K]$. This is the reason for adding the extra string. Since $x_{0}$ is centralized by $\gamma$ we can take $X=\left\langle x_{0}\right\rangle$ above and conclude that $V_{n} /(1-\gamma) V_{n}$ is the Alexander module of $\hat{g}$.

Finally, set $F_{0}=\left\langle x_{1}, x_{2}, \ldots, x_{n}\right\rangle \subseteq F_{n+1}$ and let $\hat{\gamma}$ be the link defined by $\gamma$. Then $\varphi\left(F_{0}\right)=\pi_{1}\left(\mathbb{S}^{3}-\hat{\gamma}\right)$, and if we put $K_{0}=K \cap F_{0}$, then $K_{0} / K_{0}^{\prime}\left[\gamma, F_{0}\right]$ is the Alexander module of $\hat{\gamma}$. Recall that $f_{i}=$ $x_{0} x_{i}^{-1} K^{\prime}$ and that the elements $e_{i}=f_{i}-f_{i+1}=x_{i} x_{i+1}^{-1} K^{\prime} \quad(1 \leq i<n)$ are a $\mathbb{Z}\left[t, t^{-1}\right]$-basis for $U_{n}$. It follows easily that $K_{0} K^{\prime} / K^{\prime}=U_{n}$. 
In order to keep track of the various relevant subgroups of $F$, the following lattice diagram may be helpful:

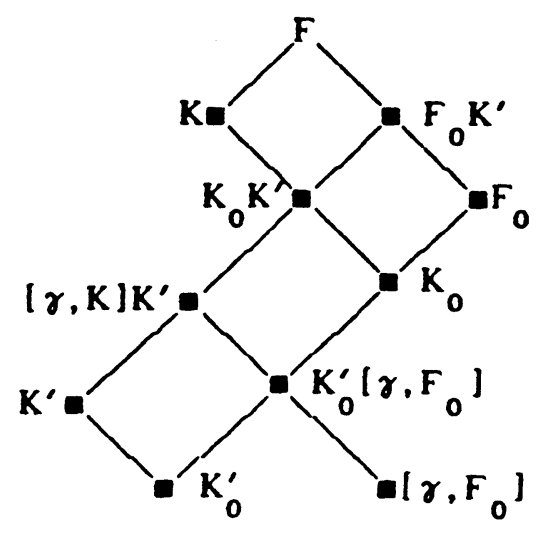

In such a diagram, a downward sloping line from $A$ to $B$ indicates that $A \supseteq B$. A parallelogram with $A$ at the top, $B$ and $C$ at the sides and $D$ at the bottom indicates that $A=B C$ and $D=B \cap C$. The above diagram makes several such assertions:

(a) $F=K F_{0}$,

(b) $\left[\gamma, F_{0}\right] \subseteq K_{0}$,

(c) $K_{0}^{\prime}=K^{\prime} \cap K_{0}$,

(d) $\left[\gamma, F_{0}\right] K^{\prime}=[\gamma, K] K^{\prime}$.

All remaining relationships are elementary group-theoretic consequences of these. Assertion (a) follows immediately from $\varepsilon\left(x_{1}\right)=1$, and (b) follows from the previously noted containment $[\gamma, F] \subseteq K$. As for (c), we have $K_{0}^{\prime} \subseteq K^{\prime} \cap K_{0}$ and, by the isomorphism theorems, $\left(K^{\prime} \cap K_{0}\right) / K_{0}^{\prime}$ is the kernel of the natural epimorphism $K_{0} / K_{0}^{\prime} \rightarrow$ $K_{0} K^{\prime} / K^{\prime}$. But both of these groups are free $R$-modules of rank $n-1$, so the natural map is an isomorphism.

To prove $(\mathrm{d})$, we already have shown that $[\gamma, K]=[\gamma, F]$, whence $\left[\gamma, F_{0}\right] K^{\prime} \subseteq[\gamma, K] K^{\prime}$. Conversely, since $K / K^{\prime}$ is generated as an $R$-module by $\left\{f_{1}, \ldots, f_{n}\right\}$, it follows that

$$
K=\left\langle x_{0}^{j}\left(x_{0} x_{i}^{-1}\right) x_{0}^{-j} \mid 1 \leq i \leq n, j \in \mathbb{Z}\right\rangle K^{\prime} .
$$

Notice that since $\left[\gamma, F_{0}\right]$ is normalized by $F_{0}$ and $\left[\gamma, F_{0}\right] K^{\prime} \unlhd K$, we get $\left[\gamma, F_{0}\right] K^{\prime} \unlhd F$ by (a). Now repeated application of $(*)$ shows that $[\gamma, K] K^{\prime}$ is contained in the normal closure in $F$ of

$$
\left\langle\left[\gamma, x_{i}^{-1}\right] \mid 1 \leq i \leq n\right\rangle K^{\prime}
$$


which is evidently contained in the normal subgroup $\left[\gamma, F_{0}\right] K^{\prime}$. It now follows from the isomorphism theorems that

$$
K_{0} / K_{0}^{\prime}\left[\gamma, F_{0}\right] \cong K_{0} K^{\prime} /[\gamma, K] K^{\prime},
$$

which says precisely that $U_{n} /(1-\gamma) V_{n}$ is the Alexander module of $\hat{\gamma}$.

4. The sesqui-linear form. Now fix $\gamma \in B_{n}$, let $R=\mathbb{Z}\left[t, t^{-1}\right]$, $W=W(\gamma)=\operatorname{im}(1-\gamma)$, and let $A=A(\gamma)$ be the complete inverse image of the $R$-torsion submodule of $V_{n} / W$. We want to define a pairing ( , ) $)_{\gamma}: A \times W \rightarrow R$ which is sesqui-linear, that is, conjugate linear (with respect to the automorphism ${ }^{*}$ ) in the first variable, and linear in the second. Set $(a, w)_{\gamma}=(a, v)$ where $v$ is any element of $(1-\gamma)^{-1}(w)$. Any two choices of $v$ differ by an element $u$ with $\gamma(u)=u$. To see that $(a, w)_{\gamma}$ is independent of the choice of $v$, choose $r \in R$ such that $r a \in W$ and let $r a=(1-\gamma)\left(v_{1}\right)$. Then

$$
r^{*}(a, u)=(r a, u)=\left(v_{1}-\gamma\left(v_{1}\right), u\right)=\left(v_{1}, u\right)-\left(v_{1}, \gamma^{-1}(u)\right)=0
$$

and therefore $(a, u)=0$. This shows that $(,)_{\gamma}$ is well defined. It is obvious that $(,)_{\gamma}$ is sesqui-linear with values in $R$.

Let $a \rightarrow \bar{a}$ be the natural map $A \rightarrow \bar{A}=A / W$, and choose $x, y \in A$. We define an element $\langle\bar{x}, \bar{y}\rangle=\langle\bar{x}, \bar{y}\rangle_{\gamma}$ of the $R$-module $\mathbb{Q}(t) / R$ as follows: choose $r \in R$ with $r y \in W$ and put $\langle\bar{x}, \bar{y}\rangle=$ $r^{-1}(x, r y)_{\gamma}+R$.

We first argue that $\langle\bar{x}, \bar{y}\rangle$ is independent of the choice of $r$. Suppose $r_{1} y \in W$. Then

$$
r_{1}^{-1}\left(x, r_{1} y\right)_{\gamma}-r^{-1}(x, r y)_{\gamma}=\frac{r\left(x, r_{1} y\right)_{\gamma}-r_{1}(x, r y)_{\gamma}}{r r_{1}}=0 .
$$

We next argue that $\langle\bar{x}, \bar{y}\rangle$ is independent of the representatives $x, y$. If $y-y_{1}=w \in W$, then

$$
r^{-1}(x, r y)_{\gamma}-r^{-1}\left(x, r y_{1}\right)_{\gamma}=r^{-1}(x, r w)_{\gamma}=(x, w)_{\gamma} \in R .
$$

On the other hand, if $x-x_{1}=(1-\gamma)(v) \in W$ and $r y=(1-\gamma)\left(v_{1}\right)$ then $-r \gamma^{-1}(y)=\left(1-\gamma^{-1}\right)\left(v_{1}\right)$, and

$$
\begin{aligned}
(x, r y)_{\gamma}-\left(x_{1}, r y\right)_{\gamma} & =\left(v-\gamma(v), v_{1}\right)=\left(v, v_{1}\right)-\left(v, \gamma^{-1}\left(v_{1}\right)\right) \\
& =-r\left(v, \gamma^{-1}(y)\right)
\end{aligned}
$$

whence $r^{-1}(x, r y)_{\gamma}-r^{-1}\left(x_{1}, r y\right)_{\gamma}=-\left(v, \gamma^{-1}(y)\right) \in R$.

It is now easily verified that $\langle,\rangle_{\gamma}$ is a sesqui-linear form on $\bar{A}(\gamma)$. To see that it is $(-t)$-Hermitian, choose $x, y \in A(\gamma)$ and $r \in R$ such that

$$
r x=u-\gamma(u), \quad r y=v-\gamma(v)
$$


for some $u, v \in V$. By expanding both sides, we easily verify the identity

$$
(r x, v)+(u, r y)=(r x, r y) .
$$

Dividing both sides by $r r^{*}$ and using $(u, y)=t(y, u)^{*}$ we have

$$
r^{-1}(x, v)+t\left[r^{-1}(y, u)\right]^{*}=(x, y)
$$

and thus

$$
\langle\bar{x}, \bar{y}\rangle+t\langle\bar{y}, \bar{x}\rangle^{*}=0 .
$$

We will show that the pair $\left(\bar{A}(\gamma),\langle,\rangle_{\gamma}\right)$ depends only on the link $\hat{\gamma}$ (up to a form-preserving isomorphism) by showing that it is invariant under the Markov moves ([1], p. 51).

There are two moves. Suppose first that $\xi \in B_{n}$. Then the identity

$$
\left(1-\xi \gamma \xi^{-1}\right)\left(V_{n}\right)=\xi(1-\gamma) \xi^{-1}\left(V_{n}\right)=\xi(1-\gamma)\left(V_{n}\right)
$$

shows that multiplication by $\xi$ induces an isomorphism $W(\gamma) \cong$ $W\left(\xi \gamma \xi^{-1}\right)$ and thus isomorphisms $\bar{V}(\gamma) \cong \bar{V}\left(\xi \gamma \xi^{-1}\right)$ and $\bar{A}(\gamma) \cong$ $\bar{A}\left(\xi \gamma \xi^{-1}\right)$. We claim that

$$
(\xi x, \xi y)_{\xi \gamma \xi^{-1}}=(x, y)_{\gamma} \quad \text { for all } x \in A(\gamma), y \in W .
$$

Namely, let $(1-\gamma)(v)=y$. Then

$$
\begin{aligned}
(\xi-\xi \gamma)(v) & =\xi y=\left(1-\xi \gamma \xi^{-1}\right)(\xi v), \\
(\xi x, \xi y)_{\xi \gamma \xi^{-1}} & =(\xi x, \xi v)=(x, v)=(x, y)_{\gamma}
\end{aligned}
$$

as required.

The second Markov move is trickier. With the standard embedding $B_{n} \subseteq B_{n+1}$ we have $B_{n+1}=\left\langle B_{n}, \sigma_{n}\right\rangle$ and we need to find a formpreserving isomorphism $\bar{A}(\gamma) \rightarrow \bar{A}\left(\sigma_{n}^{ \pm 1} \gamma\right)$. We have the inclusion $V_{n} \subseteq V_{n+1}$; in fact $V_{n+1}=V_{n} \oplus R f_{n+1}=V_{n} \oplus R e_{n}$.

LEMMA. $W\left(\sigma_{n}^{ \pm 1} \gamma\right)=W(\gamma) \oplus R e_{n}$.

Proof. We make use of the formal identity

$$
\left(1-\sigma_{n}^{ \pm 1} \gamma\right)=\left(1-\sigma_{n}^{ \pm 1}\right)+(1-\gamma)-\left(1-\sigma_{n}^{ \pm 1}\right)(1-\gamma)
$$

This implies that $W\left(\sigma_{n}^{ \pm 1} \gamma\right) \subseteq W\left(\sigma_{n}^{ \pm 1}\right)+W(\gamma)$. Note that

$$
\left(1-\sigma_{n}\right)\left(f_{i}\right)= \begin{cases}0 & \text { for } i<n, \\ t e_{n} & \text { for } i=n, \\ -e_{n} & \text { for } i=n+1\end{cases}
$$


and

$$
\left(1-\sigma_{n}^{-1}\right)\left(f_{i}\right)= \begin{cases}0 & \text { for } i<n, \\ -t^{-1} e_{n} & \text { for } i=n, \\ e_{n} & \text { for } i=n+1,\end{cases}
$$

so $W\left(\sigma_{n}^{ \pm 1}\right)=R e_{n}$. In particular, $W\left(\sigma_{n}^{ \pm 1}\right)+W(\gamma)=W\left(\sigma_{n}^{ \pm 1}\right) \oplus$ $W(\gamma)$. Moreover, $\left(1-\sigma_{n}^{ \pm 1} \gamma\right)\left(f_{n+1}\right)=\left(1-\sigma_{n}^{ \pm 1}\right)\left(f_{n+1}\right)= \pm e_{n}$ because $\gamma\left(f_{n+1}\right)=f_{n+1}$, and therefore we have $W\left(\sigma_{n}^{ \pm 1}\right) \subseteq W\left(1-\gamma \sigma_{n}^{ \pm 1}\right)$. Now $(*)$ implies that $W(\gamma) \subseteq W\left(\sigma_{n}^{ \pm 1} \gamma\right)$ and the lemma follows.

Now the inclusion map $V_{n} \subseteq V_{n+1}$ induces a map $V_{n} / W(\gamma) \subseteq$ $V_{n+1} / W(\gamma)$, and since $V_{n+1}=V_{n} \oplus R e_{n}$ the lemma implies that

$$
\frac{V_{n+1}}{W\left(\sigma_{n}^{ \pm 1} \gamma\right)}=\frac{V_{n} \oplus R e_{n}}{W(\gamma) \oplus R e_{n}} \cong \frac{V_{n}}{W(\gamma)} \text {. }
$$

Explicitly, the map $\varphi: \bar{V}(\gamma) \rightarrow \bar{V}\left(\sigma_{n}^{ \pm 1} \gamma\right)$ given by $\varphi(v+W(\gamma))=$ $v+W\left(\sigma_{n}^{ \pm 1} \gamma\right)$ is an isomorphism. We need to show that $\varphi$ is formpreserving on the torsion submodule.

We first define linear functionals $\alpha, \beta$ on $V_{n+1}$ as follows: for $x \in V_{n+1}$ write $x=x_{0}+\alpha(x) f_{n}+\beta(x) f_{n+1}$, where $x_{0} \in V_{n-1}$. We next observe that

$$
\begin{aligned}
\left(1-\sigma_{n}\right)(x) & =(\beta(x)-t \alpha(x)) e_{n}, \\
\left(1-\sigma_{n}^{-1}\right)(x) & =\left(t^{-1} \beta(x)-\alpha(x)\right) e_{n},
\end{aligned}
$$

and that if we define $S(x)=(\beta(x)-t \alpha(x)) f_{n+1}$, then

$$
\begin{gathered}
\left(1-\sigma_{n}\right) S(x)=(\beta(x)-t \alpha(x)) e_{n}=\left(1-\sigma_{n}\right)(x), \\
\left(1-\sigma_{n}^{-1}\right) S(x)=\left(t^{-1} \beta(x)-\alpha(x)\right) e_{n}=\left(1-\sigma_{n}^{-1}\right)(x) .
\end{gathered}
$$

Since $\gamma\left(f_{n+1}\right)=f_{n+1}$ it follows easily that

$$
\left(1-\sigma_{n}^{ \pm 1} \gamma\right) S(x)=\left(1-\sigma_{n}^{ \pm 1}\right)(x) \text { for all } x \in V_{n+1} .
$$

Now we get

$$
\begin{aligned}
1-\sigma_{n}^{ \pm 1} \gamma & =\left(1-\sigma_{n}^{ \pm 1}\right) \gamma+(1-\gamma) \\
& =\left(1-\sigma_{n}^{ \pm 1} \gamma\right) S \gamma+(1-\gamma)
\end{aligned}
$$

and thus

$$
1-\gamma=\left(1-\sigma_{n}^{ \pm 1} \gamma\right)(1-S \gamma)
$$

To show that $\varphi$ is form-preserving on $\bar{A}$, it suffices to show that if $a \in A(\gamma)$ and $w \in W(\gamma)$, then $(a, w)_{\gamma}=(a, w)_{\sigma_{n}^{ \pm 1} \gamma}$. Choose 
$v \in V_{n+1}$ with $(1-\gamma)(v)=w$ and set $u=(1-S \gamma)(v)$. Then $\left(1-\sigma_{n}^{ \pm 1} \gamma\right)(u)=w$, so

$$
(a, w)_{\gamma}=(a, v) \text { and }(a, w)_{\sigma_{n}^{ \pm 1} \gamma}=(a, u) .
$$

We conclude that

$$
(a, w)_{\gamma}-(a, w)_{\sigma_{n}^{ \pm 1} \gamma}=(a, v-u)=(a, S \gamma(v))=r\left(a, f_{n+1}\right)
$$

for some $r \in R$ because $\operatorname{im}(S)=R f_{n+1}$. Now choose $r_{1} \in R$ with $r_{1} a \in W$. Since $\varepsilon_{0}(\gamma(v))=\varepsilon_{0}(v)$ for all $v \in V_{n+1}, W(\gamma) \subseteq \operatorname{ker}\left(\varepsilon_{0}\right)$. Thus,

$$
r_{1} a=\sum_{i=1}^{n-1} \alpha_{i} f_{i} \quad \text { where } \sum_{i=1}^{n-1} \alpha_{i}=0 .
$$

But then

$$
r_{1}^{*}\left(a, f_{n+1}\right)=\left(r_{1} a, f_{n+1}\right)=t \sum_{i=1}^{n-1} \alpha_{i}=0
$$

and therefore $\left(a, f_{n+1}\right)=0$ as required.

To complete the proof of Theorem 2, we must show that the induced map $\bar{A} \rightarrow \operatorname{Hom}_{R}(\bar{A}, \mathbb{Q}(t) / R)$ is an isomorphism when the Alexander module is torsion. We defer this argument to the next section, where we obtain explicit formulas.

5. Computations. Theorem 1 says that a presentation for the Alexander module of a link $\hat{\gamma}$ can be obtained as follows. Let $w_{j}=$ $(1-\gamma)\left(f_{j}\right)=\sum_{i=1}^{n-1} w_{i j} e_{i} \quad(1 \leq j \leq n)$. Since $\sum t^{j-1} f_{j}$ is a fixed point for $\gamma$ we have $\sum t^{j-1} w_{j}=0$ and therefore any $n-1$ of the $w_{j}$ generate $W(\gamma)$. Hence, any $n-1$ columns of the matrix $W=w_{i j}$ are a presentation matrix for the Alexander module of $\hat{\gamma}$.

For example, let $\gamma=\left(\sigma_{1} \sigma_{2}^{-1}\right)^{2}$, so $\hat{\gamma}=4_{1}$. The matrix of $1-\gamma$ with respect to $\left\{f_{1}, f_{2}, f_{3}\right\}$ is

$$
\left[\begin{array}{ccc}
2 t-t^{2} & -t^{-1} & t^{-2}-2 t^{-1}+1 \\
t^{2}-t & 1 & -1 \\
-t & t^{-1}-1 & 2 t^{-1}-t^{-2}
\end{array}\right]
$$

Thus we get

$$
\begin{aligned}
& w_{1}=\left(2 t-t^{2}\right) e_{1}+t e_{2}, \\
& w_{2}=-t^{-1} e_{1}+\left(1-t^{-1}\right) e_{2}
\end{aligned}
$$

so $\bar{A}(\gamma)$ is generated by $\left\{\bar{e}_{1}, \bar{e}_{2}\right\}$ subject to the relations

$$
\bar{e}_{1}=(t-1) \bar{e}_{2}, \quad \bar{e}_{2}=(t-2) \bar{e}_{1},
$$


and we obtain the presentation $\bar{A}(\gamma)=\left\{\bar{e}_{1} \mid\left(t^{2}-3 t+1\right) \bar{e}_{1}=0\right\}$.

Returning to the general situation, let $W_{0}$ be a matrix consisting of any $n-1$ columns of $W$, and let $\Delta=\operatorname{det}\left(W_{0}\right)$. Evidently, $\Delta$ is the Alexander polynomial. Assuming $\Delta \neq 0$, or equivalently that $A(\gamma)=U_{n},\left\langle\bar{e}_{i}, \bar{e}_{j}\right\rangle$ can be computed by first solving the equation

$$
\Delta e_{j}=\sum_{k} u_{k j} w_{k}
$$

for $u_{k j}$. Thus, $U=u_{k j}$ is the classical adjoint of $W_{0}$, and

$$
U W_{0}=W_{0} U=\Delta I \text {. }
$$

Then

$$
\left\langle\bar{e}_{i}, \bar{e}_{j}\right\rangle=\Delta^{-1} \sum_{k} u_{k j}\left(e_{i}, f_{k}\right)=\Delta^{-1}\left[t u_{i j}-u_{i+1, j}\right] .
$$

In the above example, we get

$$
\begin{gathered}
\left(t^{2}-3 t+1\right) e_{1}=\left(t^{-1}-1\right) w_{1}+t w_{2} \\
\left\langle\bar{e}_{1}, \bar{e}_{1}\right\rangle=\Delta^{-1}\left[t\left(t^{-1}-1\right)-t\right]=\frac{1-2 t}{t^{2}-3 t+1} .
\end{gathered}
$$

Scaling by $\left(1-t^{-1}\right)$ and reducing modulo $R$, we get

$$
\left(1-t^{-1}\right)\left\langle\bar{e}_{1}, \bar{e}_{1}\right\rangle=\frac{-1}{t-3+t^{-1}} .
$$

In the general case, we put

$$
T=\left[\begin{array}{ccccc}
t & -1 & 0 & \cdots & 0 \\
0 & t & -1 & 0 & \\
& \cdot & \cdot & & \\
& & & \cdot & \\
0 & 0 & \cdots & 0 & t
\end{array}\right]
$$

and $b_{i j}=\left\langle\bar{e}_{i}, \bar{e}_{j}\right\rangle$. Then $B=\Delta^{-1} T U$. Using this formula, we can now complete the proof of Theorem 2 .

For $\bar{x}, \bar{y} \in \bar{A}$, define $\varphi_{\bar{x}}(\bar{y})=\langle\bar{x}, \bar{y}\rangle$. To show that the map $\Phi(\bar{x})=\varphi_{\bar{x}}$ is an isomorphism, we construct the inverse map as follows. Let $\varphi \in \operatorname{Hom}_{R}(\bar{A}, \mathbb{Q}(t) / R)$. Since $A=U_{n}$ is free on $\left\{e_{1}, \ldots\right.$, $\left.e_{n-1}\right\}, \varphi$ can be lifted to a map $\varphi^{\prime}: A \rightarrow \mathbb{Q}(t)$ with $\varphi(W(\gamma)) \subseteq R$. Let $\varphi^{\prime}\left(e_{i}\right)=y_{i}$ and let $y=\left(y_{1}, \ldots, y_{n-1}\right) \in \mathbb{Q}(t)^{n-1}$. Then $y W_{0} \in R$. Let $x=\left(x_{1}, \ldots, x_{n-1}\right)$ be the row vector defined by $x^{*}=y W_{0} T^{-1}$. Then $x^{*} \in R$ because $T$ is unimodular. Moreover, $x^{*} T U=y W_{0} U=\Delta y$ and thus $x^{*} B=y$. If we therefore let 
$\bar{x}=\sum x_{i} \bar{e}_{i} \in \bar{A}$, we see that $\left\langle\bar{x}, \bar{e}_{j}\right\rangle=\varphi\left(\bar{e}_{j}\right)$ for all $j$. Put $\Psi(\varphi)=\bar{x}$. Then we have shown that $\Phi(\Psi(\varphi))=\varphi$. Conversely, if we choose any $\bar{x}=\sum x_{i} \bar{e}_{i} \in \bar{A}$, set $x=\left(x_{1}, \ldots, x_{n-1}\right)$ and let $\varphi=\varphi_{\bar{x}}$, then there is an obvious lift $\varphi^{\prime}$ corresponding to the row vector $y=x^{*} B$. Then $x^{*}=y W_{0} T^{-1}$ and $\Psi\left(\varphi_{\bar{x}}\right)=\bar{x}$ as required.

6. Symmetries. Using the standard generators and relations for the braid group, it is easy to see that there is an automorphism $\gamma \rightarrow \gamma^{\prime}$ such that $\sigma_{i}^{\prime}=\sigma_{i}^{-1}$ for all $i$. Then $\hat{\gamma}^{\prime}$ is just $\hat{\gamma}$ with all crossings reversed, i.e. the mirror image. Moreover, $\gamma^{\prime-1}$ as a word in the $\sigma_{i}$ is just $\gamma$ read backwards, so $\hat{\gamma}^{\prime-1}$ is the inverse of $\hat{\gamma}$. Then $\hat{\gamma}^{-1}$ is obtained from $\hat{\gamma}$ by reversing both its orientation and the orientation of $\mathbb{S}^{3}$.

The symmetry $\gamma \rightarrow \gamma^{-1}$ is the easiest to analyze, so we will begin there. From the identity $-\gamma^{-1}(1-\gamma)=1-\gamma^{-1}$ it follows that multiplication by $-\gamma^{-1}$ induces an isomorphism $U_{n} / W(\gamma) \cong U_{n} / W\left(\gamma^{-1}\right)$. Choose $x, y \in A(\gamma), r \in R$, and $v \in V_{n}$ with $r y=(1-\gamma) v$. Then $-r \gamma^{-1} y=\left(1-\gamma^{-1}\right) v$ and since $-\gamma^{-1}$ is unitary we have

$$
\begin{aligned}
\left\langle-\gamma^{-1} \bar{x},-\gamma^{-1} \bar{y}\right\rangle_{\gamma^{-1}} & =r^{-1}\left(-\gamma^{-1} x, v\right) \\
& =-r^{-1}(x, \gamma v)=-r^{-1}(x, v-r y) .
\end{aligned}
$$

It follows that

$$
\left\langle-\gamma^{-1} \bar{x},-\gamma^{-1} \bar{y}\right\rangle_{\gamma^{-1}}=-\langle\bar{x}, \bar{y}\rangle_{\gamma}
$$

To analyze the mirror-image symmetry $\gamma \rightarrow \gamma^{\prime}$, we define a map * on elements $v \in V_{n}$ (resp. $R$-linear maps $T: V_{n} \rightarrow V_{n}$ ) by applying the ring automorphism ${ }^{*}$ to each co-ordinate of $v$ (resp. matrix entry of $T$ ) with respect to the basis $\left\{f_{1}, f_{2}, \ldots, f_{n}\right\}$. Then $(T v+w)^{*}=$ $T^{*} v^{*}+w^{*}$, and $\left(T_{1} T_{2}\right)^{*}=T_{1}^{*} T_{2}^{*}$. For $\gamma \in B_{n}$ we abuse notation slightly by writing $\gamma^{*}$ for the action of the conjugate Burau matrix.

Define $P: V_{n} \rightarrow V_{n}$ via $P\left(f_{i}\right)=f_{n-i-1}$. Then $P^{*}=P^{-1}=P$. Moreover, from the definition of the Squier form we have $\left(P f_{i}, P f_{j}\right)$ $=\left(f_{j}, f_{i}\right)$ from which it follows by sesqui-linearity that

$$
(P x, P y)=\left(y^{*}, x^{*}\right) \text { for all } x, y \in V_{n} \text {. }
$$

Define $\delta_{1}=\sigma_{1}$, and inductively set $\delta_{i+1}=\sigma_{1} \sigma_{2} \cdots \sigma_{i} \delta_{i}$. Then $\delta_{n}$ is a half-twist of all $n+1$ strings, and it is easily checked that $\delta_{n} \sigma_{i} \delta_{n}^{-1}=$ $\sigma_{n-i}(1 \leq i<n)$. By inspecting matrix entries we verify that

$$
\delta P \sigma_{i}^{*} P^{-1} \delta^{-1}=\sigma_{i}^{-1} \quad(1 \leq i<n)
$$


from which we obtain the basic identity

$$
\delta P \gamma^{*} P^{-1} \delta^{-1}=\gamma^{\prime} \text { for all } \gamma \in B_{n} .
$$

Now choose $y, v \in V_{n}$ and $r \in R$ with $r y=(1-\gamma) v$. Then

$$
\begin{aligned}
r^{*} y^{*}=\left(1-\gamma^{*}\right) v^{*} & =(\delta P)^{-1}\left(1-\gamma^{\prime}\right) \delta P\left(v^{*}\right), \quad \text { and thus } \\
r^{*} \delta P\left(y^{*}\right) & =\left(1-\gamma^{\prime}\right) \delta P\left(v^{*}\right) .
\end{aligned}
$$

It follows that the map $y \mapsto y^{\prime}=\delta P\left(y^{*}\right)$ defines a (conjugate-linear) isomorphism $U_{n} / W(\gamma) \rightarrow U_{n} / W\left(\gamma^{\prime}\right)$. Moreover, we have

$$
\begin{aligned}
\left\langle x^{\prime}, y^{\prime}\right\rangle_{\gamma^{\prime}} & =\left(\delta P x^{*}, \delta P v^{*}\right) / r^{*}=\left(P x^{*}, P v^{*}\right) / r^{*}=(v, x) / r^{*} \\
& =t(x, v)^{*} / r^{*}=t\langle x, y\rangle_{\gamma}^{*} .
\end{aligned}
$$

This result together with equation $(*)$ implies that for the inverse symmetry $\gamma \rightarrow \gamma^{\prime-1}$, the map $y \mapsto y^{\prime \prime}=-\gamma^{-1} \delta P\left(y^{*}\right)$ defines a conjugate linear isomorphism $U_{n} / W(\gamma) \rightarrow U_{n} / W\left(\gamma^{\prime-1}\right)$ with

$$
\left\langle x^{\prime \prime}, y^{\prime \prime}\right\rangle_{\gamma^{\prime-1}}=-t\langle x, y\rangle_{\gamma}^{*} \text {. }
$$

These results have particularly simple consequences in the special case that the Alexander module is cyclic with generator $e$ and annihilator $\Delta$. The form is completely determined by the element $\langle e, e\rangle$, and $e^{\prime}$ is another generator iff $e^{\prime}=\alpha e$ for some unit $\alpha$ of $R / \Delta R$. Since the form is $(-t)$-hermitian, we have $\langle e, e\rangle=-t\langle e, e\rangle^{*}$ which easily implies that non-invertibility cannot be detected in this case. If, however, $\hat{\gamma}$ is amphicheiral, then for some unit $\alpha$ of $R / \Delta R$ we have

$$
\alpha \alpha^{*}\langle e, e\rangle=t\langle e, e\rangle^{*} \text {. }
$$

Non-singularity of the form implies that $\langle e, e\rangle$ is a unit, and thus we get the necessary condition $\alpha \alpha^{*}=-1$ for some unit $\alpha$.

7. Taking the trace. We first observe that the form $\langle$,$\rangle actually$ takes values in a cyclic submodule of $\mathbb{Q}(t) / R$ isomorphic to $R / R r$ for some $r \in R$. Namely, recall that $R$ is a noetherian UFD and $V_{n} / W$ is finitely generated. Thus, $\bar{A}$ has a finite set of generators $\left\{\bar{a}_{1}, \ldots, \bar{a}_{m}\right\}$. Let $r=\operatorname{lcm}\left\{\operatorname{ann}_{R}\left(\bar{a}_{i}\right)\right\}$. Then the form takes values in the $R$-module $R r^{-1} / R$ which is isomorphic to $R / R r$. Alternatively, we could take $r$ to be the Alexander polynomial of $\gamma$.

We can assume that the gcd of the coefficients of $r$ is 1 (this amounts to the assertion that $V_{n} / W$ is $\mathbb{Z}$-torsion free, which can be easily seen by specializing the Burau representation at $t=1$ ). Tensoring with $\mathbb{Q}$ then embeds $R / R r$ into the finite dimensional algebra $\mathbb{Q}\left[t, t^{-1}\right] /(r)$ which admits the canonical linear functional $\operatorname{tr}_{(r)}$, the 
trace of the regular representation. By applying this functional, we obtain a rational valued form $\langle,\rangle_{0}=\operatorname{tr}_{(r)}\langle$,$\rangle on the Alexander$ module. This form appears to depend on the choice of denominator $r$, but in fact it does not. For if we replace $r$ by $r s$, the coset representing a given value $\langle\bar{x}, \bar{y}\rangle$ of the form is also multiplied by $s$ and thus has zero trace on the submodule $(r) /(r s)$ of $\mathbb{Q}\left[t, t^{-1}\right] /(r s)$. Since the isomorphism

$$
\frac{\mathbb{Q}\left[t, t^{-1}\right] /(r s)}{(r) /(r s)} \cong \mathbb{Q}\left[t, t^{-1}\right] /(r)
$$

implies that $\operatorname{tr}_{(r s)}=\operatorname{tr}_{(r)}+t^{\prime}$ where $t^{\prime}(x)$ is the trace of the restriction of $\operatorname{ad}(x)$ to $(r s) /(r),\langle,\rangle_{0}$ does not depend on $r$.

For example, in the calculation for the figure eight knot, we got the hermitian

$$
\left(1-t^{-1}\right)\left\langle\bar{e}_{1}, \bar{e}_{1}\right\rangle=\frac{-1}{t-3+t^{-1}} .
$$

since $t-3+t^{-1}$ is monic, the Alexander module is finitely generated over $\mathbb{Z}$, in this case it is $\mathbb{Z} \oplus \mathbb{Z}$. If we take the basis $\left\{e_{1}, t e_{1}\right\}$, the action of $t$ is $\left[\begin{array}{cc}0 & -1 \\ 1 & 3\end{array}\right]$ and the matrix of the trace form is $\left[\begin{array}{ll}-2 & -3 \\ -3 & -2\end{array}\right]$.

8. The $(n, m)$ torus link. Let $\tau=\sigma_{1} \sigma_{2} \cdots \sigma_{n-1}$. Then the obvious braid representative for the $(n, m)$ torus link is $\gamma=\tau^{m}$. It is easy to see that

$$
\begin{aligned}
\tau\left(f_{i}\right) & =(1-t) f_{1}+t f_{i+1} \quad(1 \leq i<n), \\
\tau\left(f_{n}\right) & =f_{1} .
\end{aligned}
$$

Let $e_{i}=f_{i}-f_{i+1} \quad(1 \leq i<n)$ as above. We will calculate with respect to the basis $\left\{e_{1}, e_{2}, \ldots, e_{n-1}, f_{n}\right\}$ of $V_{n}$. Define $e_{0}=f_{n}-f_{1}$ and note that $e_{0}=-\sum_{i=1}^{n-1} e_{i}$. It is easily checked that $\tau\left(e_{i}\right)=t e_{i+1}$ for all $i$ with subscripts modulo $n$. Moreover, $\tau\left(f_{n}\right)=f_{1}=f_{n}-e_{0}$, so we have

$$
\tau^{m}\left(e_{i}\right)=t^{m} e_{i+m}, \quad \tau^{m}\left(f_{n}\right)=f_{n}-\sum_{i=0}^{m-1} t^{i} e_{i} .
$$

The Alexander module $A_{n m}$ for the $(n, m)$ torus link is therefore generated by $\left\{\bar{e}_{0}, \bar{e}_{1}, \ldots, \bar{e}_{n-1}\right\}$ subject to the relations
(1) $\bar{e}_{i+m}=t^{-m} \bar{e}_{i} \quad(0 \leq i<n)$,
(2) $\sum_{i=0}^{n-1} \bar{e}_{i}=0$,
(3) $\sum_{i=0}^{m-1} t^{i} \bar{e}_{i}=0$.

These relations may be unraveled as follows. Let $d=\operatorname{gcd}(n, m)$, $m=k d, n=l d$, and $e=\operatorname{lcm}(n, m)$. The relations (1) can be 
iterated to obtain

$$
\bar{e}_{i+r m}=t^{-r m} \bar{e}_{i} \quad(0 \leq i<n)
$$

for any integer $r$. Note that there are exactly $l$ distinct multiples of $m$ modulo $n$, namely $\{0, d, 2 d, \ldots,(l-1) d\}$ and a set of orbit representatives for translation by $m$ is given by $\{0,1, \ldots, d-1\}$. Thus, relations (1) say precisely that $A_{n m}$ is generated by $\left\{\bar{e}_{0}, \bar{e}_{1}, \ldots, \bar{e}_{d-1}\right\}$ and that $\left(1-t^{l m}\right) \bar{e}_{i}=0 \quad(0 \leq i<d)$. Since $l m=k n=e$, we see that $\left(1-t^{e}\right)$ annihilates $A_{n m}$. Relations (2) and (3) can be re-written in terms of $\left\{\bar{e}_{0}, \bar{e}_{1}, \ldots, \bar{e}_{d-1}\right\}$ as follows. Let $a$ be the least positive integer such that $a m \equiv d(\bmod n)$. Then $\bar{e}_{i+d}=t^{-a m} \bar{e}_{i}$ for all $i$. Let

$$
\begin{aligned}
& u=\bar{e}_{0}+\bar{e}_{1}+\cdots+\bar{e}_{d-1}, \\
& v=\bar{e}_{0}+t \bar{e}_{1}+\cdots+t^{d-1} \bar{e}_{d-1} .
\end{aligned}
$$

Then relation (2) says that

$$
t^{-(l-1) a m}\left(1+t^{a m}+t^{2 a m}+\cdots+t^{(l-1) a m}\right) u=0
$$

and relation (3) becomes

$$
\left(1+t^{d-a m}+t^{2(d-a m)}+\cdots+t^{(k-1)(d-a m)}\right) v=0 .
$$

Let $d-a m=b n$, and put

$$
p_{m}(t)=\frac{1-t^{l a m}}{1-t^{a m}}=\frac{1-t^{a e}}{1-t^{a m}}, \quad p_{n}(t)=\frac{1-t^{k b n}}{1-t^{b n}}=\frac{1-t^{b e}}{1-t^{b n}} .
$$

There are two special cases to consider: $a=0, b=1$, in which case $d=n$; and $a=1, b=0$, in which case $d=m$. In the former case, put $p_{m}(t)=1$, and in the latter case, put $p_{n}(t)=1$. Then is all cases, we can re-write (4) and (5) as

$$
p_{m}(t) u=0=p_{n}(t) v
$$

However, we also have $\left(1-t^{e}\right) u=0=\left(1-t^{e}\right) v$, so $u$ (resp. $v$ ) is annihilated by the gcd of $p_{m}(t)$ (resp. $\left.p_{n}(t)\right)$ and $1-t^{e}$. Now, $p_{m}(t)$ is the product of the cyclotomic polynomials $\Phi_{r}(t)$ as $r$ ranges over all divisors of $a e=a m l$ which do not divide $a m$. Since $d=a m+b n$, we have $1=a k+b l$ which implies that if $r \mid e$ and $r \mid a m$, then $r \mid m$. We conclude that

$$
\frac{1-t^{e}}{1-t^{m}} u=0=\frac{1-t^{e}}{1-t^{n}} v
$$


It is not difficult now to check that $A_{n m}$ is generated by $\left\{\overline{\boldsymbol{e}}_{0}, \bar{e}_{1}, \ldots\right.$, $\left.\bar{e}_{d-1}\right\}$ subject to the relations (6) and

$$
\left(1-t^{e}\right) \bar{e}_{i}=0 \quad(0 \leq i<d) .
$$

To present $A_{n m}$ as a direct sum of cyclic modules, we set

$$
\begin{aligned}
& v^{\prime}=\frac{1}{1-t}(u-v)=\sum_{i=1}^{d-1} \frac{1-t^{i}}{1-t} \bar{e}_{i}, \quad \text { and } \\
& u^{\prime}=\frac{1-t^{b n}}{1-t^{d}} u+t^{b n} \frac{1-t^{a m}}{1-t^{d}} v=\frac{1-t^{b n}}{1-t^{d}} u+\left[1-\frac{1-t^{b n}}{1-t^{d}}\right] v .
\end{aligned}
$$

Routine calculations then show that $\left\{u^{\prime}, v^{\prime}, \bar{e}_{2}, \ldots, \bar{e}_{d-1}\right\}$ is a basis for $A_{n m}$, provided that $d \geq 2$ and $d \neq n, m$. Moreover, it follows that

$$
\frac{\left(1-t^{e}\right)\left(1-t^{d}\right)}{\left(1-t^{n}\right)\left(1-t^{m}\right)} u^{\prime}=0=\frac{\left(1-t^{e}\right)(1-t)}{\left(1-t^{d}\right)} v^{\prime},
$$

and that relations (7) and (8) imply (6) and (7).

Note that in the knot case $(d=1)$ we have $v=u=u^{\prime}=\bar{e}_{0}$. Hence, the Alexander module is cyclic with annihilator

$$
\left(1-t^{n m}\right)(1-t) /\left(1-t^{n}\right)\left(1-t^{m}\right) \text {. }
$$

If either $n \mid m$ or $m \mid n$ then $u^{\prime}=0$ and $\left\{v^{\prime}, \bar{e}_{2}, \ldots, \bar{e}_{d-1}\right\}$ is a basis for $A_{n m}$. For example, $A_{2,2 k}$ is cyclic with annihilator

$$
\left(1-t^{2 k}\right)(1-t) /\left(1-t^{2}\right) \text {. }
$$

Finally, if $n=m$, then $u^{\prime}=v^{\prime}=0$ and $\left\{\bar{e}_{2}, \ldots, \bar{e}_{d-1}\right\}$ is a basis.

To evaluate $\left\langle\bar{e}_{i}, \bar{e}_{j}\right\rangle$ we use relations $(1)$ to write

$$
\left(1-t^{e}\right) e_{j}=\left(1-\tau^{m}\right)\left(e_{j}+t^{m} e_{j+m}+t^{2 m} e_{j+2 m}+\cdots+t^{(l-1) m} e_{j+(l-1) m}\right) .
$$

Hence we obtain

$$
\left\langle\bar{e}_{i}, \bar{e}_{j}\right\rangle=\frac{1}{1-t^{e}} \sum_{p=0}^{l-1} t^{p m}\left(e_{i}, e_{j+p m}\right) .
$$

Recall that

$$
\left(e_{i}, e_{j}\right)=\left\{\begin{array}{ll}
1+t & \text { if } i=j \\
-t & \text { if } i=j-1 \\
-1 & \text { if } i=j+1 \\
0 & \text { if }|i-j|>1
\end{array} \quad \text { for } 1 \leq i, j<n\right.
$$


In fact, the same formulas extend to $0 \leq i, j<n$, and using them, it is not difficult to make $(*)$ explicit. For example, in the knot case we get

$$
\left\langle\bar{e}_{0}, \bar{e}_{0}\right\rangle=\frac{1}{1-t^{n m}}\left[1+t-t^{a m}-t^{1-a m}\right]=\frac{\left(1-t^{a m}\right)\left(1-t^{b n}\right)}{1-t^{n m}} .
$$

\section{REFERENCES}

[1] J. Birman, Braids, Links, and Mapping Class Groups., Annals of Mathematics Studies 85, Princeton Univ. Press, 1975.

[2] R. C. Blanchfield, Intersection theory of manifolds with operators with applications to knot theory, Ann. of Math., 65 (1957), 340-356.

[3] C. Squier, The Burau representation is unitary, Proc. Amer. Math. Soc., 90 (1984), 199-202.

[4] H. F. Trotter, On S-equivalence of Seifert matrices, Inventiones Math., 20 (1973), 173-207.

Received April 22, 1988 and in revised form March 3, 1989. Thanks are due to Andrew Casson and Vaughan Jones for many helpful discussions on the present topic.

UNIVERSITY OF CALIFORNIA

BERKELEY, CA 94720

AND

CENTER For COMMUNications Research

PRINCETON, NJ 08540 


\section{PACIFIC JOURNAL OF MATHEMATICS EDITORS}

\author{
V. S. VARADARAJAN \\ (Managing Editor) \\ University of California \\ Los Angeles, CA 90024-1555-05 \\ Herbert Clemens \\ University of Utah \\ Salt Lake City, UT 84112 \\ THOMAS ENRIGHT \\ University of California, San Diego \\ La Jolla, CA 92093
}

R. FINN

Stanford University

Stanford, CA 94305

Hermann Flaschka

University of Arizona

Tucson, AZ 85721

VAUGHAN F. R. Jones

University of California

Berkeley, CA 94720

Steven Kerckhoff

Stanford University

Stanford, CA 94305

\author{
C. C. MOORE \\ University of California \\ Berkeley, CA 94720
}

MARTIN SCHARLEMANN

University of California

Santa Barbara, CA 93106

HAROLd STARK

University of California, San Diego

La Jolla, CA 92093

\section{ASSOCIATE EDITORS}
R. ARENS
E. F. BECKENBACH
B. H. NeUmanN
F. WoLF
(1904-1989)
K. YosHIDA
(1906-1982)

\section{SUPPORTING INSTITUTIONS}

UNIVERSITY OF ARIZONA
UNIVERSITY OF BRITISH COLUMBIA
CALIFORNIA INSTITUTE OF TECHNOLOGY
UNIVERSITY OF CALIFORNIA
MONTANA STATE UNIVERSITY
UNIVERSITY OF NEVADA, RENO
NEW MEXICO STATE UNIVERSITY
OREGON STATE UNIVERSITY

UNIVERSITY OF ARIZONA

CAIVERSITY OF BRITISH COLUN UNIVERSITY OF CALIFORNIA

MONTANA STATE UNIVERSITY OREGON STATE UNIVERSITY

\author{
UNIVERSITY OF OREGON \\ UNIVERSITY OF SOUTHERN CALIFORNIA \\ STANFORD UNIVERSITY \\ UNIVERSITY OF HAWAII \\ UNIVERSITY OF TOKYO \\ UNIVERSITY OF UTAH \\ WASHINGTON STATE UNIVERSITY \\ UNIVERSITY OF WASHINGTON
}

The Supporting Institutions listed above contribute to the cost of publication of this Journal, but they are not owners or publishers and have no responsibility for its content or policies.

Mathematical papers intended for publication in the Pacific Journal of Mathematics should be in typed form or offset-reproduced (not dittoed), double spaced with large margins. Please do not use built up fractions in the text of the manuscript. However, you may use them in the displayed equations. Underline Greek letters in red, German in green, and script in blue. The first paragraph must be capable of being used separately as a synopsis of the entire paper. In particular it should contain no bibliographic references. Please propose a heading for the odd numbered pages of less than 35 characters. Manuscripts, in triplicate, may be sent to any one of the editors. Please classify according to the 1980 Mathematics Subject Classification (1985 Revision) scheme which can be found in the December index volumes of Mathematical Reviews. Supply name and address of author to whom proofs should be sent. All other communications should be addressed to the managing editor, or Elaine Barth, University of California, Los Angeles, California 90024-1555-05.

There are page-charges associated with articles appearing in the Pacific Journal of Mathematics. These charges are expected to be paid by the author's University, Government Agency or Company. If the author or authors do not have access to such Institutional support these charges are waived. Single authors will receive 50 free reprints; joint authors will receive a total of 100 free reprints. Additional copies may be obtained at cost in multiples of 50 .

The Pacific Journal of Mathematics (ISSN 0030-8730) is published monthly. Regular subscription rate: $\$ 190.00$ a year (12 issues). Special rate: $\$ 95.00$ a year to individual members of supporting institutions.

Subscriptions, orders for numbers issued in the last three calendar years, and changes of address should be sent to Pacific Journal of Mathematics, P.O. Box 969, Carmel Valley, CA 93924, U.S.A. Old back numbers obtainable from Kraus Periodicals Co., Route 100, Millwood, NY 10546.

The Pacific Journal of Mathematics at P.O. Box 969, Carmel Valley, CA 93924 (ISSN 0030-8730) is published monthly. Second-class postage paid at Carmel Valley, California 93924, and additional mailing offices. Postmaster: send address changes to Pacific Journal of Mathematics, P.O. Box 969, Carmel Valley, CA 93924.

\section{PUBLISHED BY PACIFIC JOURNAL OF MATHEMATICS, A NON-PROFIT CORPORATION}




\section{Pacific Journal of Mathematics}

Vol. 144, No. $2 \quad$ June, 1990

George E. Andrews and David M. Jackson, An algebraically derived $q$-analogue of a character sum associated with a class of semiregular

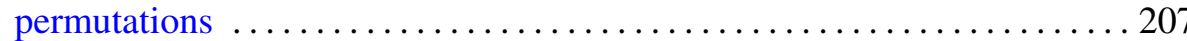

Fabio Bardelli and Andrea Del Centina, The moduli space of genus four double covers of elliptic curves is rational $\ldots \ldots \ldots \ldots \ldots \ldots \ldots \ldots 219$

Young Do Chai, An estimate of the volume of a compact set in terms of its

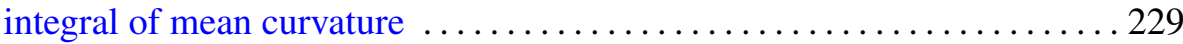

Salvador Comalada, Elliptic curves with trivial conductor over quadratic fields

Kahar El-Hussein, Résolubilité semi-globale des opérateurs différentiels invariants sur les groupes de déplacements $\ldots \ldots \ldots \ldots \ldots \ldots \ldots \ldots . \ldots 259$

David M. Goldschmidt, Classical link invariants and the Burau representation

Liliana Janicka, Radon-Nikodým problem for the variation of a vector measure

Wacław Marzantowicz, An almost classification of compact Lie groups with Borsuk-Ulam properties ............................ 299

Akira Ohbuchi, On the projective normality of some varieties of degree 5 . . 313 Ken'ichi Ohshika, Minimal measured laminations in geometric

3-manifolds

Hal Leslie Smith, A discrete Lyapunov function for a class of linear differential equations

John Samuel Spielberg, Diagonal states on $O_{2} \ldots \ldots \ldots \ldots \ldots \ldots \ldots \ldots$

Thomas Vogel, A note on the sessile drop

Gerold Wagner, On means of distances on the surface of a sphere (lower bounds) 